\title{
Del género epistolar a las publicaciones científicas periódicas de acceso abierto
}

\section{From epistolary literature to open access scientific periodic publications}

\author{
Eugenio Matijasevic. Bogotá, D.C. (Colombia)
}

El género epistolar cambió para siempre con el advenimiento de la Internet y su Protocolo para Transferencia Simple de Correo (Simple Mail Transfer Protocol o SMTP). Este protocolo de red, asociado al Protocolo de Oficina Postal (Post Office Protocol, en la actualidad POP3) o al Protocolo de Acceso a Mensajes de Internet (Internet Message Access Protocol, en la actualidad IMAP4) permite enviar cartas desde una máquina electrónica a un servidor de tal manera que el destinatario las pueda recuperar del servidor desde otra máquina electrónica. La máquina electrónica en cuestión puede ser un ordenador de escritorio, un ordenador portátil, una tableta electrónica o un "teléfono inteligente" (sea lo que sea lo que el adjetivo quiera significar en este caso). Paulatinamente hemos sustituido el sustantivo "carta" por el de correo electrónico, e-mail o, simplemente, "mail", reservando "carta" para documentos de carácter más oficial y burocrático (cartas credenciales, carta de emplazamiento, carta de despido, carta pastoral, carta de recomendación, carta de renuncia).

Con la aparición del correo electrónico otras tecnologías de la comunicación (el correo postal, la telegrafía, el correo aéreo, el télex, el fax), quedaron ipso facto obsoletas como tecnologías de la comunicación por escrito a distancia. Siguen siendo útiles en muchas otras funciones, por ejemplo para el envío de paquetes por correo, cosa que, por fortuna, aún no podemos hacer a través de las máquinas electrónicas y la Internet, mientras no se ponga a punto la invención de la teletransportación prefigurada en la serie Star Trek.

En lo que respecta a la comunicación escrita, el correo electrónico con su rapidez, facilidad de empleo y accesibilidad (limitada obviamente a la franja de la población que puede pagar por tener acceso a una máquina electrónica que incluya correo), ha hecho que la utilización de otros medios de comunicación escrita a distancia haya venido menguando de manera paulatina, dando por descontado que la posibilidad de comunicarse a distancia de viva voz, gracias primero a la telefonía y luego a la telefonía móvil, ya había hecho gran parte del trabajo.

¿Alguien recuerda la última vez que envió o recibió un telegrama?. En Colombia 4-72, el servicio oficial de correos del país, sigue enviando telegramas desde cualquiera de sus puntos de venta, expendios y aliados en todos los municipios del país (1) pero, en general, este servicio sólo se utiliza para asuntos judiciales y cobros y se mantiene gracias a la necesidad de que quede constancia documentada del envío y la recepción del documento y porque es obligatorio para todas las instituciones oficiales (incluidos los juzgados), de acuerdo con el artículo 43 del Decreto 229 de 1995, utilizar los servicios de 4-72 excluyendo otras empresa de correos (2). En otros países la telegrafía ha desaparecido por completo y no es posible ya enviar un telegrama. En Australia, por ejemplo, si se empeña uno en buscar la manera de enviar un telegrama y accede a la página de Australia Post la búsqueda por Telegram lo llevará a una página de glosarios en donde podremos enterarnos de que Telegraphy/Telegram se refiere a "An old way of communicating where a message could be sent over long distances by electrical currents or Morse code. The message was often called a telegram" (Una antigua manera de comunicarse por medio de la cual se podía enviar un mensaje a través de grandes distancias mediante corrientes
Dr. Eugenio Matijasevic: Editor General Acta Médica Colombiana. Bogotá, D.C. (Colombia). E-mail: eugenio.matijasevic@gmail.com Recibido: 26/IV/2016 Aceptado: 28/IV/2016 
eléctricas o código Morse. El mensaje, con frecuencia, era denominado un telegrama) (3). En la India la empresa estatal BSNL suspendió el servicio de telegrafía a partir del 15 de julio de 2013 (4). En Estados Unidos de América la Western Union, la compañía a cargo de la telegrafía en ese país por más de 160 años, cerró el servicio de telegrafía el 27 de enero de 2006 vendiendo lo poco que quedaba de ese renglón de la empresa a iTelegram, un enclenque servicio de telegrafía dedicado a enviar telegramas en ocasiones especiales (bautismos, bodas, funerales) que garantiza rapidez y entrega personal también para cancelación de contratos (5).

¿Alguien recuerda cuándo fue la última vez que tuvo que utilizar un sello de correos? Obviamente aún los necesitamos para el envío de documentos y paquetes, aunque cada vez más han sido sustituidos por un timbre impreso directamente sobre el paquete por una máquina selladora. Además, ya el correo británico (Royal Mail [6]), el correo de los Estados Unidos de América (USPS [7]) y el correo australiano (Australia Post [8]) permiten pagar a través de internet, con el número de una tarjeta de crédito, el importe de correos e imprimir un sello de correos por el valor necesario, incluso con una imagen personalizada, para colocarlo sobre el paquete que se va a enviar. Dinamarca y Suecia ya no tienen sellos de correos: desde 2011 basta obtener un código de envío a través de un mensaje telefónico de texto y anotar en el sobre o paquete a enviar el código recibido (9).

¿Cuántas tarjetas postales envió este año? ¿Cuántas recibió? Compare la cifra que da respuesta a las preguntas anteriores con la que da respuesta a las siguientes ¿Cuantas fotografías envió a través del correo electrónico o incluso mediante nuevas aplicaciones como whatsapp o iMessage desde diferentes lugares del mundo para mostrarles, no sólo contarles, a sus familiares y amigos en donde se encontraba? ¿Cuántas recibió?. La mayoría de las personas menores de 25 años a las que he interrogado al respecto no tiene idea de qué es una tarjeta postal.

No sólo han desaparecido o tienden a desaparecer antiguas maneras de comunicación, como dice del telegrama el Australia Post, sino que el crecimiento exponencial del correo electrónico ha traído aberraciones sin fin. No es que antaño no existieran los fraudes cometidos a través del correo (también se pueden cometer delitos por carta, o intentar cometerlos) y tampoco es posible asegurar que no existiera el correo basura (los buzones o los apartados postales o los apartados aéreos repletos de cartas publicitarias que el destinatario nunca había solicitado), pero no existían a la escala que es posible que se den ahora gracias precisamente a la velocidad y posibilidad de reduplicación que brinda el correo electrónico. El spam es un nombre nuevo, antaño no era posible enviar un millón de cartas publicitarias a una base de datos de direcciones ofreciendo un determinado producto porque los costos de impresión y de envío hacían ese tipo de publicidad ineficiente por lo costosa, el correo electrónico ha hecho que dichos costos sean insignificantes, basta tener la base de datos y el resto del trabajo es fácil y barato a costa de la irritación de los destinatarios. Originalmente spam era el nombre de una comida enlatada norteamericana producida por Hormel Foods a base de "Spiced Pork and hAM", pero terminó por significar cualquier forma de abuso en la red, en especial correo basura (junk e-mail), gracias a una escena de un programa humorístico de televisión del grupo Monty Python en la serie Monty Python's Flying Circus en la que en un restaurante de mala muerte todos los platos del menú están acompañados de spam (incluso spam con spam y spam con spam con spam) y la camarera repite la palabreja una y otra vez, hasta el cansancio, mientras anuncia el menú a los comensales. La similitud es clara: spam es algo que se repite y se repite hasta la irritación, pero ¿cómo terminó el spam del restaurante de los Monty Python aplicándose al correo electrónico masivo, repetitivo y abusivo? Esa parte de la historia la relata de manera muy detallada Brad Templeton en su página de Internet (10).

En diversas ocasiones diversos escritores de ficción han escrito novelas cuya trama se enreda y desenreda a partir de intercambios epistolares entre los diversos personajes, dichas "Novelas Epistolares" se basan en la ficción de que se trata de cartas reales. La primerísima de todas en la literatura universal fue la Cárcel de Amor de Diego de San Pedro (11), que sienta las bases de un género en el que han incursionado incluso autores recientes. Entre ellos Thornton Wilder con The Ides of March (12), publicada en 1948, de la que hay una excelente traducción al castellano en Alianza Editorial: Los Idus de Marzo (13), considerada por García Márquez una de las mejores obras de ficción ("hermosa novela", "manantial de belleza", "fuente deslumbrante de la grandeza y las miserias del poder", "[la] leí por primera vez hace unos 25 años [...] y [la] he releído muchas veces desde entonces con el mismo placer" [14]) o el premio Nobel de Literatura Saul Bellow con Herzog (15) considerada por muchos (entre ellos Harold Bloom) la mejor obra de Bellow y una de las mejores cien novelas de habla inglesa publicadas desde 1923 (16).

Recientemente, cómo podría no haber ocurrido, se han publicado algunas novelas epistolares basadas en la ficción de que se trata de correos electrónicos reales y realmente intercambiados. The Lawgiver de Herman Wouk (17) (el mismo del Motín del Caine [18] y Vientos de Guerra [19]) me sirve para citar sólo un ejemplo de verdadera calidad de "novela epistolar electrónica" (o a base de e-mails).

A pesar de esta y otras obras literarias epistolares electrónicas, quizás porque los correos son fingidos, meros recursos literarios, sólo artificios para ayudar a la trama, no puedo dejar de pensar que la literatura universal aún está a la espera de una colección de e-mails escritos dentro de los cánones del verdadero género epistolar, similar a las Epístolas Morales a Lucilio de Séneca (20) o a las Cartas a Ático (21) o las Cartas a su Hermano Quinto (22) de Cicerón o las Cartas de Plinio el Joven (23). No creo, sinceramente, que de los miles de millones de correos electrónicos que 
duermen en la nube se pueda hacer algo similar. Una gran parte de la correspondencia por $e$-mail (a pesar de las leyes contra el spam) es correo basura, solicitudes no solicitadas para ingresar a redes sociales a las que no pertenezco y a las que no quiero pertenecer (glosando a Groucho Marx: no me gustaría pertenecer a ninguna red social que me admitiera como miembro [24]), publicidad no pedida sobre píldoras mágicas para adelgazar o sobre secretos ancestrales para aumentar la longitud de ciertas partes de la anatomía, solicitudes de ricas herederas africanas que necesitan nuestra ayuda para recuperar las fortunas perdidas de su padre banquero muerto en extrañas circunstancias en el extranjero o instrucciones sobre cómo reclamar el premio de un millón de libras esterlinas de una supuesta lotería electrónica cuyo boleto nunca compramos o a la que nunca nos suscribimos pero que el azar determinó que nuestra dirección de correo electrónico fuera la ganadora -junto con 20 millones de direcciones más-.

En síntesis, la aparición de la Internet ha permitido, con respecto a las comunicaciones por escrito que llamábamos cartas, acelerar de manera antaño inimaginable la velocidad de intercambio, pero ha incrementado los problemas (correo basura, intentos de fraude) sin haber producido hasta el momento nada que pudiera llamarse un género literario, algo que implique un aporte al crecimiento y desarrollo cultural de la humanidad en su conjunto, por el contrario ha conseguido volver cada vez más trivial y anodina la comunicación escrita, aunque indudablemente ha permitido acelerar el intercambio de información en lo referente a toda clase de negocios.

Los efectos del desarrollo de Internet sobre las publicaciones científicas periódicas son también, como en el caso del género epistolar (de las cartas escritas en hojas de metal de la antigüedad clásica al e-mail), de doble carácter: aunque dicho desarrollo ha traído inmensas ventajas (rapidez, accesibilidad, reproducibilidad y un largo etcétera), ha traído también, como se verá, inconvenientes a los que el sector de las publicaciones científicas apenas está aprendiendo a adaptarse.

Cada desarrollo tecnológico trae aparejados, tarde que temprano, cambios en las costumbres y en los asuntos humanos que le incumben. Antes de la aparición de la imprenta, las comunicaciones científicas necesariamente debían hacerse mediante cartas, cartas que muchas veces eran públicas y circulaban de mano en mano hasta que alguien que las había leído quería una copia para sí y acudía a los copistas o escribas que las copiaban una y otra vez con los consabidos errores que se transmitirían a las siguientes copias. La correspondencia científica puede remontarse hasta la antigüedad clásica. En épocas tan remotas como los siglos quinto y cuarto AEC se conocieron colecciones de cartas, muchas de ellas de contenido científico y filosófico, de Platón, Aristóteles y Epicuro, aunque tal vez sólo algunas del segundo podrían considerarse verdaderas comunicaciones científicas (25). Con el desarrollo de la imprenta surgió la posibilidad de copiar las cartas científicas y de hacer numerosas copias exactamente iguales sin los errores acumulativos de los copistas. Se hizo entonces posible otra forma de comunicación escrita de los hallazgos científicos diferente a la correspondencia, y se hizo concreta la idea genial de Henry Oldemburg de establecer una publicación científica periódica impresa, The Philosophical Transactions, dedicada a la difusión de los resultados de las investigaciones de las personas dedicadas a la Philosophia Naturalis, nombre del que deriva el título de la revista y con el que entonces se conocía lo que en la actualidad denominamos simplemente ciencia. La revista acaba de cumplir 351 años de publicación ininterrumpida (26) y no sólo fue la primera publicación científica periódica sino la primera en emplear el arbitraje por pares de cada artículo que iba a ser publicado (27).

El hecho es que, antes de la imprenta, la idea de Oldemburg hubiese sido impensable: habría que imaginar una publicación científica periódica hecha a mano por un impagable ejército de copistas. Con el tiempo, sin embargo, se requirió para el manejo integral de la producción de las publicaciones científicas periódicas toda la parafernalia, esta sí pagable, de las empresas editoriales encargadas de la impresión, distribución, promoción, publicidad, etc. de las publicaciones científicas periódicas, que, cómo no, se vieron obligadas a ingresar al mercado capitalista como cualquier otra mercancía, dependiendo de las leyes de la oferta y la demanda y de la mano invisible del mercado de la que hablara Smith (28).

En la actualidad, con el advenimiento de la Internet, es posible hacer publicaciones científicas periódicas con innumerables copias exactas de las mismas y distribuirlas a cualquier lugar del mundo en el que haya una máquina electrónica conectada a la red. Pero hacer dicha publicación tiene un costo, incluso aunque con la distribución electrónica se esté haciendo un inmenso ahorro en papel, imprenta y envío por correo.

De alguna manera, a medida que las publicaciones científicas periódicas se volvieron (cada vez más) digitales, los sistemas previos de suscripción periódica se tornaron obsoletos pues, de hecho, las suscripciones empezaron a caer gracias a la posibilidad de muchos lectores de acceder al contenido digital de la revista a través de la biblioteca de su universidad sin necesidad de caminar hasta ella o simplemente pidiéndole a un amigo que le enviase por $e$ mail la copia en PDF del artículo que necesitaba. El precio de las publicaciones científicas periódicas digitales, por su parte, se disparó, quizás como una medida de los gerentes de las empresas editoriales de compensar un poco las pérdidas en suscripciones y limitar el acceso directo a los artículos científicos: si usted está realizando un trabajo de investigación y requiere con urgencia consultar trabajos similares publicados en revistas a las que no está subscrito podrá acceder al repositorio de la empresa editorial que las publica, pero no podrá obtener los artículos sin pagar 
antes mediante su tarjeta de crédito entre 20 y 40 dólares por cada uno.

Sin embargo la presión creciente de grupos de científicos y de usuarios y de activistas de la Internet con respecto al libre acceso a las publicaciones científicas en la red, como una manera de impulsar el desarrollo de las ciencias gracias al libre intercambio de publicaciones científicas periódicas, ha llevado a que se intente hacer algo para cambiar el modelo. Este movimiento se ha aglutinado alrededor del grupo Open Access, un conglomerado de editores de publicaciones científicas periódicas, directores de institutos de investigación, decanos de universidades, directores de academias científicas, directores de bibliotecas y de bases de datos, etc., que, en la Iniciativa de Budapest (Budapest Open Access Initiative [29] firmada el 14 de Febrero de 2002), la Declaración de Bethesda (Bethesda Statement on Open Access Publishing [30] firmada el 20 de junio de 2003) y la Declaración de Berlín (Berlin Declaration on Open Access to Knowledge in the Sciences and Humanities [31] firmada el 22 de octubre de 2003), aboga por el libre acceso a toda la información científica sin ningún tipo de obstáculo, sobre todo sin el obstáculo del pago de suscripciones.

La definición más precisa de Open Acces, basada en las definiciones del llamado panel BBB (por Budapest, Bethesda y Berlín) es, sin duda, la que propone Peter Suber en su libro Open Access. Para él, acceso abierto (open access) a la literatura científica es "su libre disponibilidad en la Internet pública, que permita a cualquier usuario leer, descargar, copiar, distribuir, imprimir, buscar y enlazar los textos íntegros de estos artículos, recorrerlos para indexación, pasarlos como datos a software o utilizarlos para cualquier otro propósito legal; sin que existan barreras financieras, legales o técnicas, diferentes a aquellas inseparables del proceso de tener acceso a la red de Internet. La única limitación a la reproducción y a la distribución, y el único papel de los derechos de propiedad intelectual en este campo, sería dar a los autores control sobre la integridad de su trabajo y el derecho a ser adecuadamente reconocidos y citados" (32).

Como era de esperarse, la iniciativa de Open Access ha sido recibida con una clara oposición por parte de las empresas editoriales aglutinadas alrededor de The International Association for Scientific, Technical and Medical Publishers (STM). No es para menos, pues de cualquier manera el libre acceso a las publicaciones científicas tal como lo concibe Open Access significaría el final de su negocio.

Los partidarios del Open Acces insisten en que, cuando se ha realizado una investigación y esta va a ser publicada, la sociedad en su conjunto ha pagado ya demasiado por ella (a través de subvenciones a las universidades o a los investigadores mediante becas gubernamentales o mediante los mismos sueldos que reciben de la universidad) como para pensar que la sociedad tenga que volver a pagar por la difusión de los resultados de la investigación. STM, por su parte, advierte que los costos de publicación y de difusión de la investigación científica son muy altos y que la única forma de garantizar la calidad de lo que se publica es a través de la intermediación de las empresas editoriales y de "la inversión que han hecho ellas durante décadas en una infraestructura abierta, mundial y poderosa para la amplia diseminación de los resultados de la investigación" (33).

Algunos se refieren a las empresas editoriales que defienden el modelo opuesto al Open Access como Toll-Access publishers (empresas editoriales de revistas de acceso con barreras o de acceso pagando peaje), pero los gerentes de dichas empresas editoriales prefieren el término más neutral y menos peyorativo de conventional publishers (empresas editoriales de revistas convencionales) (34).

La discusión entre los partidarios de Open Access y los partidarios de STM va para largo. Algunos gobiernos, entre ellos los de las dos más grandes potencias mundiales en investigación, Estados Unidos de América (35) y China (36), se han alineado, aunque sólo parcialmente, del lado de Open Access, declarando que los investigadores que hayan recibido subsidios gubernamentales están en la obligación de hacer accesible su investigación a través de una plataforma de acceso libre. Ambos gobiernos, sin embargo, han hecho concesiones a las empresas editoriales asociadas en STM aceptando un periodo de espera de hasta un año entre el momento de publicación del artículo y el momento de archivarlo en una base de datos de acceso libre ("embargo" es el término que utiliza STM para referirse a esta práctica) dado que, en opinión de las empresas, la clave del éxito de la transición a Open Access sigue siendo la sostenibilidad, y dicha sostenibilidad sólo puede garantizarse, según dichas empresas, mediante la adición de los fondos monetarios necesarios para mantenerla o mediante periodos de embargo que permitan que el modelo de suscripciones continúe operando (37).

La tesis de que ya la sociedad pagó por la difusión de la información científica desde el momento en el que financió dicha investigación, no convence a nadie que dirija una empresa editorial. En un modelo socialista de producción a lo mejor se llegaría al consenso de compensar económicamente a las personas encargadas de la difusión del conocimiento ya producido, pero en el modelo capitalista imperante, desde el editor en jefe hasta los correctores de textos preguntarán de dónde van a salir los fondos para compensar económicamente su trabajo, cómo se va a financiar la publicación. La pregunta es válida en el concierto económico actual.

La mayoría de las publicaciones científicas periódicas que nacieron en la era de Internet y son Open Access han encontrado el cómo basándose en una lógica muy simple que ya se había empleado en Gran Bretaña con motivo de la gran reforma postal de 1840. Hasta ese año el costo del envío de correspondencia era pagado siempre por el destinatario en el momento en que la carta llegaba a sus manos, y dicho costo dependía de las millas de distancia entre su dirección y la del remitente. En síntesis, el pago se hacía después de realizado el trabajo de llevar la carta 
hasta el destinatario, pero este muchas veces no tenía con qué (o no quería) pagar los costos de envío y la carta era regresada al remitente quien tampoco estaba dispuesto a pagar por una entrega de correspondencia que no se había realizado. Obviamente, el rendimiento del proceso era pobre y las devoluciones incrementaban aún más los costos haciendo el sistema ineficiente. Hasta que las autoridades aceptaron oír las propuestas de Rowland Hill, quien había escrito unos años antes un opúsculo al respecto: Post Office reform, its Importance and Practicability, cuya publicación había tenido que pagar de su propio bolsillo. La propuesta era simple: el costo de envío de la correspondencia no sería por millas sino por peso (hasta una onza, más o menos dos hojas de papel, valdría un chelín), no importaría la distancia entre remitente y destinatario (Hill había calculado muy bien que la mayor parte de los costos reales del servicio postal Británico se debían a gastos generales, estructurales al sistema mismo, y que los costos marginales de enviar una carta a un destino muy lejano eran más bien bajos y se compensarían si, como en efecto ocurrió, se incrementaba el número de usuarios del sistema) y, finalmente y el punto fundamental, el costo de envío de la correspondencia debería ser pagado por adelantado por el remitente, con lo que, aunque el destinatario no recibiera o no quisiera recibir la correspondencia, el trabajo de hacerla llegar a su destino ya estaba pago. Además, como constancia del prepago del envío, encima del sobre de la carta se colocaría el otro invento de Hill: una etiqueta engomada autoadhesiva con el valor del importe pagado (había nacido el sello postal o estampilla) (38). Sobra anotar que el éxito de la reforma postal de Hill fue tal que en los años siguientes el resto de servicios postales del mundo se acogió a la reforma Británica.

Así pues, las publicaciones científicas periódicas nuevas, que querían crecer rápidamente en la red y ser consultadas de manera fácil y sin trabas, se acogieron rápidamente a las propuestas del Open Access y encontraron en la antigua propuesta de Rowland Hill la manera de mantenerse a flote en una economía de mercado que aparentemente no estaba hecha para el acceso abierto. Es por ello que en muchas revistas Open Access no es el lector (el destinatario en el modelo de Hill) quien paga por la revista o por el artículo científico que desea leer, sino que quien paga por el artículo es el investigador, el que ha realizado una investigación y ha escrito un artículo científico describiendo sus hallazgos (el remitente en el modelo de Hill). Una vez que el artículo enviado ha sido aceptado para publicación, el autor o autores deben desembolsar una suma de dinero, calculada por la revista y hecha explícita de antemano, correspondiente a lo que denominan APC (Article processing charges: gastos por procesamiento del artículo).

Acogerse a este modelo ha traído enormes ventajas. No es otra la razón del éxito de Public Library of Science (PLoS) que nació hace apenas quince años y publica siete revistas, todas arbitradas por pares, todas de altísima ca- lidad, todas con los máximos indicadores de lectura y de citación: PLoS One, PLoS Biology, PLoS Medicine, PLoS Computational Biology, PLoS Genetics, PLoS Pathogens y PLoS Neglected Tropical Diseases (39). No es otra la razón del éxito de BioMed Central, una empresa editorial que nació hace también 15 años y en la actualidad publica 290 revistas científicas, todas ellas arbitradas por pares (40). Algunas de ellas son open peer review, una práctica alabada por unos, denostada por otros, en la que los nombres de los autores son conocidos por los pares y los de los pares por los autores, al tiempo que el proceso de evaluación es público, abierto y transparente a través de la Internet. Al respecto, The British Medical Journal, la cuarta revista de medicina general más citada del mundo, utiliza el open peer review desde septiembre de 2014 y ha puesto a disposición de sus lectores en la Internet la historia del proceso de revisión por pares que ha llevado a la publicación de todos los artículos publicados desde entonces con el fin de mejorar la transparencia, garantizar que los pares evaluadores declaren sus conflictos de intereses de la misma manera que lo hacen los autores y hacer más abiertamente responsables a los editores y pares evaluadores del proceso de publicación (41).

La publicación de un artículo aceptado por PLoS Medicine tiene unos APC de 2900 dólares. Si el autor no tiene el dinero, no debe preocuparse, es posible una rebaja o una exención de pago si hace una solicitud formal al respecto. Algo parecido puede suceder con BioMed Central en donde el autor sin fondos puede solicitar una rebaja o, a lo mejor, la institución a la que pertenezca forme parte del Membership Program al que ya se encuentran inscritas más de 500 instituciones que pagan por adelantado y de manera global lo que consideran que serán los APC de sus investigadores. También cabe la posibilidad de que eximan del pago de los APC al autor si demuestra que su país es un país pobre, pero para el caso de Colombia no es posible alegar dicho motivo de exención pues consideran pobre al país con un GDP (Gross Domestic Product, un parámetro económico que se basa en el valor de todos los bienes y servicios producidos a lo largo de un determinado periodo, generalmente de un año) menor a 200000 millones de dólares (Colombia está por encima de ese rango de acuerdo con el Fondo Monetario Internacional [42]). Por otro lado, cada vez será más raro que un autor no tenga cómo pagar los ATC, a medida que más agencias de financiación o de subvención de investigaciones aceptan que el autor incluya dentro de los gastos de la investigación un ítem para el pago de la publicación en Open Access.

Algunas de las publicaciones que nacieron antes de la era de Internet están haciendo la transición a Open Access con un modelo mix to en el que los autores que lo deseen pueden pagar el valor del Open Access para que su artículo esté disponible para todos los lectores, incluso aquellos que no estén suscritos a la revista. Otras publicaciones, como es el caso de The British Medical Journal, han hecho obligatorio el pago de APC para todos los artículos de investigación 
(bajo el acápite research en la página de Internet de la revista [43]) y, en consecuencia, dichos artículos son Open Access pues sus autores pagaron 3000 libras antes de la publicación, en el momento en que el artículo fue aprobado para publicación, con el fin de que todos los lectores de la revista en Internet tuvieran acceso a él; pero, paradójicamente, todos los artículos solicitados por la revista (revisiones de tema bajo el acápite clinical reviews o práctica clínica bajo el acápite practice) y todos los artículos escritos por miembros de la redacción (bajo el acápite News \& Views) e incluso los editoriales, no son Openn Access y quien quiera leerlos o bien paga la suscripción a la revista o bien compra cada artículo por 23 libras esterlinas o 37 dólares o 30 euros. Son artículos Toll-Access.

En resumen: el modelo de negocios en la edición y difusión de publicaciones científicas periódicas ha cambiado y seguirá cambiando impulsado por las fuerzas del mercado hasta adaptarse, encontrando el nicho económico que mejor le permita sobrevivir al interior de las nuevas tecnologías de difusión de la información científica. Si el desarrollo de la imprenta generó cambios en dicha difusión ¿por qué no iba a generarlos el desarrollo de la Internet?. Cada vez más, las publicaciones científicas periódicas se sumarán al movimiento de Open Access y cada vez más las empresas editoriales con ánimo de lucro, e incluso algunas que no lo son, acudirán al cobro de APC para subvencionar sus gastos, haciendo que sea el remitente y no el destinatario de la información científica quien pague por su difusión. Hasta el momento, el modelo funciona. Tendremos APC para rato, mientras no surja un modelo nuevo (cabe imaginar una gran asociación mundial, por ejemplo, liderada por la ONU o por la UNESCO o por la OMS, que se encargue de recibir de los diferentes gobiernos o agencias de investigación fondos destinados a la difusión de investigación y de distribuirlos con base en algún tipo de proporción - ¿el impact factor?- a las empresas editoriales). De todas maneras, independientemente de cómo se vaya a pagar, disgústele a quien le disguste, el Open Access llegó para quedarse.

Tanta maravilla, sin embargo, no está exenta de problemas. Uno de los más apremiantes tiene que ver con una nueva clase de spam que muchos de ustedes habrán encontrado (y continuarán encontrando) en sus correos electrónicos con el ofrecimiento muy oportuno de que una determinada revista (¿cómo se enterarían de que yo estaba escribiendo un trabajo de investigación?) está en condiciones de publicar Open Access mi trabajo. Con seguridad, no se trata de ninguna revista de BioMed central o de PLoS o del grupo editorial de British Medical Journal. Pero el nombre suena conocido, se publica en inglés, se publica en el exterior, tiene un repositorio en el que el artículo estará visible para todo aquel que quiera leerlo, es arbitrada por pares, menciona estar indexada en varios índices de citación y los nombra por sus siglas agregando a su lado un factor de impacto que no es malo; además, dará un certificado de publicación para mostrar en la universidad y mejorar el escalafón (y el sueldo)... ¿por qué no?

La realidad es que se trata no sólo de otra forma de spam sino, además, de un verdadero fraude: el nombre que suena conocido no es más que eso, no es una revista conocida sino una revista con un nombre que suena conocido (¿qué tal Journal of Advances in Internal Medicine? o, mejor aún ¿Journal of Evidence Based Medicine and Healthcare?), es cierto que se publica en inglés (tal vez le manden al autor una copia en PDF de la publicación, pero sólo existe esa copia y el inglés del que hacen gala en la página de Internet es un inglés desastroso, plagado de errores ortográficos y de horrores de redacción), es cierto que se publica en el exterior (casi siempre en alguna ciudad intermedia de la India o del sureste asiático o de Nigeria aunque figuren con direcciones de Internet radicadas en Estados Unidos de América, Canadá o Europa), el repositorio no es visitado por nadie excepto, quizás, por el autor del artículo (y la base de datos desaparecerá muy pronto, una vez haya cumplido su efímera función de captar incautos, para no tener que pagar más tiempo de hosting en la red), la evaluación por pares es espuria, rápidamente aprobarán el artículo (puesto que lo que buscan es cobrar los APC), si uno mira con detalle los índices bibliográficos de citaciones en los que afirma estar indexada la revista encuentra siglas extrañas parecidas a ISI (Institute for Scientific Information), SCI (Science Citation Index) o Scopus, pero que no son ISI, SCI ni Scopus (y si alguno de ellos aparece, la mención es falsa y los valores de los factores de impacto mencionados son también falsos). Ahora bien, es posible que sí envíen un certificado de publicación, pero de nada servirá puesto que la revista no aparece indexada en ninguna parte o, peor aún, sí está indexada, pero en la lista de Jeffrey Beall.

Jeffrey Beall es un bibliotecólogo experto en metadatos y recuperación de información que ha investigado y publicado en estos campos durante los últimos 25 años y trabaja actualmente como profesor asociado y bibliotecario en la Biblioteca Auraria de la Universidad de Colorado en Denver (44). Desde enero de 2010 mantiene una página en Internet, a la que llamó Scholarly Open Acces, en la que publica datos sobre las revistas (y sobre las empresas editoriales que las publican) que más que de baja calidad se caracterizan por su deshonestidad explotando incautos en el negocio de las publicaciones científicas periódicas Open Access con prepago de APC, sin que finalmente aporten ninguno de los servicios que las verdaderas revistas Open Access dan a quienes les envían artículos para publicación: arbitraje por pares, una correcta indexación en un indice bibliográfico de citaciones conocido, un repositorio Open Access que garantice continuidad en donde todo el mundo pueda consultar el artículo y una certificación de publicación válida. En síntesis, engañan al investigador, quien habrá pagado APC por la publicación de su artículo en una revista prácticamente inexistente, sin verdadera evaluación por pares y sin figuración en parte alguna. 
Beall acuñó el término predatory publishing (empresas editoriales predadoras) para referirse a este tipo de publicaciones periódicas Open Access de baja calidad y deshonestas y renueva día a día en su página de Internet una lista de lo que llama List of Potential, possible, or probable predatory scholarly open-access publishers (45) y otra lista de lo que denomina List of Standalone Journals: Potential, possible, or probable predatory scholarly open-access journals (46). En la primera figuran empresas editoriales, cada una con un promedio de cinco revistas, que Beall considera podrían estar dedicadas a explotar la buena fe de los investigadores (había 1009 empresas de este tipo en la lista a mediados de abril de 2016). En la segunda lista figuran revistas sin empresa editorial dedicadas al mismo negocio posiblemente de manera deshonesta (había 1018 revistas de este tipo en la lista a mediados de abril de 2016). Ahora bien, la frontera entre una revista Open Acces de baja calidad y un negocio criminal montado sobre el Open Access para aprovecharse de sus fragilidades y esquilmar incautos puede ser bien difusa. Beall admite que una revista recién creada y con un soporte académico no muy rico (editores y pares académicos no muy conocidos, respaldo institucional por parte de universidades o sociedades científicas de bajo perfil) bien podría quedar clasificada en su grupo de predatory journals. Por ello Beall insiste en el título de ambas listas que los elementos que figuran en ellas son sólo potenciales, posibles o probables predatory journals, y está siempre dispuesto a revisar y revertir cualquier inclusión que el editor de la revista considere injusta. Para ello elige un grupo anónimo de revisores integrado por cuatro miembros a quienes remite cualquier apelación con el fin de que sean ellos quienes externamente le aconsejen si mantener o excluir de la lista a la revista que apela (47). Y aunque aconseja a las universidades y a las instituciones que financian investigación que no excluyan de los beneficios de publicación (escalafón universitario, por ejemplo) a los investigadores que publiquen en ellas y más bien se evalúe cada caso de manera individual y acorde a consideraciones geoeconómicas regionales, por lo general, si una revista figura en su lista, dichos beneficios son denegados.

Los criterios que utiliza Beall para incluir una revista o una empresa editorial en el grupo de predadores han sido hecho públicos con el fin de mejorar la transparencia del proceso y disminuir la controversia que ha rodeado su lista. Son criterios bastante complejos pero certeros. Se basan, en parte en el papel de los editores de las revistas (¿figuran al mismo tiempo como propietarios de la empresa editorial? ¿figuran como editores de todas las revistas que posee la empresa en lugar de que haya un editor para cada una de ellas? ¿las credenciales académicas de los editores no son muy claras?), del comité editorial (¿figuran sus miembros en más de un comité editorial del mismo conglomerado de revistas? ¿Sus credenciales académicas no son muy claras? ¿son sólo dos o tres los miembros del comité editorial?), en el tipo de negocio y su transparencia, en la honestidad de la información que aportan (por ejemplo mencionar factores de impacto falsos o indexaciones falsas) (48) y en una larga serie de datos bastante fundamentados que apuntan, aunque no concluyen, que se trata de una revista predadora. Es realmente un trabajo laborioso, que implica horas y horas de búsquedas en Internet y que, a pesar de su laboriosidad, puede fallar e incluir entre los predadores revistas nuevas de baja calidad con laxitud en la evaluación por pares. Beall lo admite, pero considera que para una revista erróneamente incluida en su lista este hecho puede ser también un acicate para mejorar su calidad (si la calidad de los procesos implícitos en la publicación de trabajos científicos en una revista es tan baja que la revista puede ser confundida con una revista predadora - "dime con quien andas y te diré quien eres", decían los antiguos- lo mejor que puede hacer dicha revista es mejorar la calidad de los mismos y apelar después para conseguir la exclusión de la lista).

Existe una manera menos laboriosa que los criterios de Beall de descubrir revistas predadoras: enviar a muchas revistas Open Access un mismo artículo con tantas fallas metodológicas que cualquier revista seria lo rechazaría y declarar predadoras a las revistas que lo acepten para publicación e intenten cobrar los APC. Eso exactamente fue lo que hizo John Bohannon, un periodista de la revista Science. Utilizando el falso nombre de Ocorrafoo Cobange, un supuesto biólogo del inexistente Wassee Institute of Medicine en Asmara, la capital de Eritrea, envió a 304 revistas Open Access los resultados de un supuesto estudio en el que describía las propiedades anticancerígenas de un producto químico que, afirmaba el tal Cobange, había logrado extraer de un liquen (49). En palabras de Bohannon, cualquier par evaluador, con apenas un poco más de los conocimientos de química impartidos en el bachillerato y con la capacidad para entender un gráfico de datos básico, se habría dado cuenta de inmediato de las deficiencias del artículo y de cómo los incorregibles defectos de los experimentos descritos implicaban que los supuestos resultados no tenían ningún sentido, por lo que habría rechazado sin apelación su publicación en la revista. La metodología de Bohannon implicaba que cualquier revista que aceptara el falso artículo no tenía un adecuado sistema de arbitraje por pares y, por tanto, estaba más interesada en recibir el dinero proveniente de los ACP que en publicar estudios verdaderamente científicos, auto-delatándose de inmediato como una revista predadora. De las 304 revistas a las que envió el artículo 29 no respondieron nunca, por lo que las declaró abandonadas por sus propietarios, 20 escribieron diciendo que el artículo aún estaba en revisión ocho meses después de enviado, 98 rechazaron el artículo y 157 lo aceptaron. De estas últimas en el 60\% de los casos no hubo ninguna evidencia de arbitraje por pares. Al comparar sus resultados con la lista de Beall, Bohannon encontró que los criterios utilizados por aquel para detectar revistas predadoras son bastante buenos: de las revistas que figuraban en la lista de Beall $82 \%$ aceptaron publicar el trabajo del supuesto 
Cobange. Pero esto también significa, dice Bohannon, que muchas revistas "predadoras" bajo los criterios de Beall, el $18 \%$ para ser exactos, no lo son realmente: hicieron un adecuado trabajo de arbitraje por pares, detectaron las fallas del trabajo y rechazaron su publicación aunque con ello se perdieran el cobro de los APC.

Son muchas las publicaciones serias que se han interesado en el tema. Nature publicó en 2013, en una edición temática sobre las transformaciones que están ocurriendo en el mundo de las publicaciones científicas periódicas, un artículo sobre la actividad desarrollada por Beall, sus implicaciones, riesgos y fortunas, que incluye la "lista de chequeo" de Butler para identificar empresas editoriales serias o confiables (A checklist to identify reputable publishers) (50) que vale la pena tener en cuenta al pensar a donde enviar para publicación un trabajo de investigación, sobre todo porque quizás resulte más fácil identificar una buena revista que detectar un predador. Annals of Internal Medicine publicó a comienzos de este año un artículo en la sección Ideas and Opinions denominado Stop Predatory Publishers Now: Act Collaboratively (51) sobre el cual me llamó la atención el Dr. Rodolfo Dennis, presidente del Comité Editorial de Acta Médica Colombiana. Los autores del artículo de Annals saben de lo que hablan y, ante todo, llaman la atención sobre la necesidad de emprender medidas inmediatas para detener o reducir al mínimo este flagelo. Las acciones no son sencillas y requieren el trabajo conjunto de todos cuantos estamos inmersos en la actividad de publicar trabajos científicos, en especial a nivel de grupos internacionales como la Asociación Mundial de Editores Médicos (World Association of Medical Editors) o la Coalición para Recursos Responsables para Publicación (Coalition for Responsible Publication Resources). De las tareas recomendadas por los autores para combatir este flagelo hay una parte más fácil de realizar: educar e investigar. Educar a los investigadores en formación e incluso a los ya formados sobre los riesgos de publicar en revistas no acreditadas e investigar (el conocimiento libera) cómo funciona el negocio de las revistas predadoras, dónde tienen sus feudos, a quiénes afecta, a dónde van los recursos financieros que acaparan, etc. Al respecto, un trabajo reciente, publicado en el Journal of the Association for Information Science and Technology demuestra mediante una tecnología fácil de replicar que quienes publican en revistas predadoras son, en su mayoría, investigadores jóvenes e inexpertos provenientes de países en vías desarrollo (52).

¿Desearía publicar su trabajo de investigación en una publicación periódica internacional en lugar de enviarlo a Acta Médica Colombiana?

\section{Referencias}

1. Telegrama. 4-72 [Internet]. Consultado el 13 de marzo de 2016. Disponible en http://www.4-72.com.co/Telegrama

2. El Servicio de Envíos de Colombia. 4-72 [Internet]. Consultado el 13 de marzo de 2016. Disponible en http://www.4-72.com.co/PreguntasFrecuentes

3. Australia Post [Internet]. Consultado el 13 de marzo de 2016. Disponible en http://auspost.com.au/education/ourpost/students/postie-kate/glossary.html

4. Sengupta S. Farewell Telegram: An era died with the last message. En DNA India Disponible en http://www.dnaindia.com/analysis/column-farewell-telegram-anera-died-with-the-last-message-1861137. Consultado el 14 de marzo de 2016.

5. iTelegram [Internet]. Consultado el 13 de marzo de 2016. Disponible en https:// itelegram.com/telegram/Western_Union_iTelegram_2006.asp

6. Royal Mail [Internet]. Consultado el 13 de marzo de 2016. Disponible en http:// www.royalmail.com/corporate/services/online-postage

7. USPS [Internet]. Consultado el 13 de marzo de 2016. Disponible en https:// es.usps.com/ship/online-shipping.htm

8. Australia Post [Internet]. Consultado el 13 de marzo de 2016. Disponible en http:// auspost.com.au/parcels-mail/personalised-stamps.html?ilink=mm-personalisedstamps-1

9. Redacción BBC. El adiós a los sellos postales [Internet]. BBC Mundo, 10 de marzo de 2011. Disponible en http://www.bbc.com/mundo/noticias/2011/03/110310 sellos sms dinamarca dfp.shtml. Consultado el 14 de marzo de 2016.

10. Templeton B. Origin of the term SPAM to mean net abuse [Internet]. Disponible en http://www.templetons.com/brad/spamterm.html. Consultado el 13 de marzo de 2016.

11. San Pedro, Diego de. Cárcel de Amor. Parrilla, Carmen (Editora). Deyermond, Alan (Estudio Preliminar). Barcelona: Editorial Crítica; 1995: 187 pp.

12. Wilder, Thornton. The Ides of March. New York: Harper Perennial; 2003: 304 pp.

13. Wilder, Thornton. Los Idus de Marzo. Madrid: Alianza Editorial; 1974: 253 pp.

14. García Marquez, Gabriel. Los Idus de Marzo. En: Notas de Prensa: Obra Periodística 5 (1961-1984). Madrid: Penguin Random House Grupo Editorial; 2015. Formato Digital iBooks.

15. Bellow, Saul. Herzog. New York: Penguin Books; 2003: 400 pp.

16. Grossman L, Lacayo R. All-TIME 100 Novels [Internet]. Cosnultado el 13 de marzo de 2016. Disponible en http://entertainment.time.com/2005/10/16/ all-time-100-novels/slide/all/

17. Wouk, Herman. The Lawgiver. New York: Simon \& Schuster; 2013: 240 pp. 18. Wouk, Herman. El motín del Caine. Barcelona: Martínez Roca; 1973: 698 pp.

19. Wouk, Herman. Vientos de Guerra. Barcelona: Editorial Grijalbo; 1984: 963 pp.

20. Seneca. Epístolas Morales a Lucilio. Dos Volúmenes. Madrid: Editorial Gredos; 2005.

21. Cicerón, Marco Tulio. Cartas a Ático. Dos volúmenes. Madrid: Editorial Gredos; 1996.

22. Cicerón, Marco Tulio. Correspondencia con su hermano Quinto, incluido el breve manual de campaña electoral. Madrid:: Alianza editorial; 2009: 232 pp.

23. Plinio el Joven. Cartas. Madrid: Editorial Gredos; 2005: 598 pp.

24. Marx, Groucho. Groucho and Me. Boston: Da Capo Press; 1995: p 321.

25. Ebbeler J. Tradition, Innovation, and Epistolary Mores. En Rousseau P (Editor): A companion to late antiquity. Willey-Blackwell; 2009: pp 270-284.

26. Philosophical Transactions, the world's 1rst science journal [Internet]. Consultado el 3 de marzo de 2016. Disponible en http://rstl.royalsocietypublishing.org/

27. Matijasevic E. Leibniz y Newton: la inercia de la soberbia. Acta Med Colomb 2010; 35 (4): 157-165

28.Smith, Adam. The Theory of Moral Sentiments. Boston: Mobile Reference. Formato Digital Kindle.

29. Budapest Open Access Initiative [Internet]. Consultada el 13 de marzo de 2016 Disponible en http://www.budapestopenaccessinitiative.org/read

30. Bethesda Statement on Open Access Publishing [Internet]. Consultada el 13 de marzo de 2016. Disponible en http://legacy.earlham.edu/ peters/fos/bethesda. htm

31. Berlin Declaration on Open Access to Knowledge in the Sciences and Humanities. [Internet]. Consultada el 13 de marzo de 2016. Disponible en http://openaccess. mpg.de/Berlin-Declaration

32. Suber, Peter. Open Access. Cambridge, Massachusetts: The MIT Press; 2012: p 7. Disponible como Open Access en https://mitpress.mit.edu/sites/default/ files/9780262517638_Open_Access_PDF_Version.pdf

33. STM News Release, 08/04/2016 [Internet]. STM response to Amsterdam Call for Action on Open Science. Consultado el 9 de abril de 2016. Disponible en http:// www.stm-assoc.org/2016 0408 News Release STM response to Call for Action on Open Science.pdf

34. Suber, Peter. Open Access. Cambridge, Massachusetts: The MIT Press; 2012 : p 6. Disponible como Open Access en https://mitpress.mit.edu/sites/default/ files/9780262517638_Open_Access_PDF_Version.pdf

35. Van Noorden R. US science to be open to all. Nature/News, 28 February 2013. doi:10.1038/494414a. Consultado el 13 de marzo de 2016. Disponible en http:// 
www.nature.com/news/us-science-to-be-open-to-all-1.12512

36. Van Noorden R. Chinese agencies announce open-access policies. Nature/ News,19 May 2014. doi:10.1038/nature.2014.15255. Consultado el 13 de marzo de 2016. Disponible en http://www.nature.com/news/chinese-agencies-announceopen-access-policies-1.15255

37. Mabe M. STM [Internet]. Extended feedback on draft Amsterdam Call for Action. Consultado el 15 de abril de 2016. Disponible en http://www.stm-assoc. org/2016 0414 STM ExtendedFeedback.pdf

38. West, Chris. A history of Britain in Thirty-six postage Stamps. London: Square Peg; 2012: pp 1-8.

39.PLoS [Internet]. Consultado el 13 de marzo de 2016. Disponible en https://www. plos.org/publications

40. BioMed Central [Internet]. Consultado el 13 de marzo de 2016. Disponible en https://www.biomedcentral.com

41. Groves T, Loder E. Prepublication histories and open peer review at The BMJ. BMJ 2014;349:g5394. Consultado el 13 de marzo de 2016. Disponible en http:// www.bmj.com/content/349/bmj.g5394

42. International Monetary Found. Report for Selected Countries and Subjects [Internet]. Consultado el 12 de abril de 2016. Disponible en http://www.imf.org/ external/index.htm

43. The British Medical Journal [Internet]. Consultado el 13 de marzo de 2016. Disponible en http://www.bmj.com/thebmj.

44. About the Author. Scholarly Open Access [Internet]. Consultado el 15 de marzo de 2016. Disponible en https://scholarlyoa.com/about/
45. Beall J. List of Potential, possible, or probable predatory scholarly open-access publishers [Internet]. Consultado el 14 de abril de 2016. Disponible en https:// scholarlyoa.com/publishers/

46. Beall J. List of Standalone Journals: Potential, possible, or probable predatory scholarly open-access journals [Internet]. Consultado el 14 de abril de 2016. Disponible en https://scholarlyoa.com/individual-journals/

47. Beall J. List of Standalone Journals: Potential, possible, or probable predatory scholarly open-access journals [Internet]. Consultado el 14 de abril de 2016. Disponible en https://scholarlyoa.com/other-pages/appeals/

48. Beall J. Criteria for Determining Predatory Open-Access Publishers (2nd edition). Consultado el 15 de marzo de 2016. Disponible en https://scholarlyoa. com/2012/11/30/criteria-for-determining-predatory-open-access-publishers-2ndedition/

49. Bohannon J. Who's Afraid of Peer Review? Science 2013; 342 (6154): 60-65. DOI: $10.1126 /$ science. 342.6154 .60

50. Butler D. Investigating journals: The dark side of publishing. Nature 49528 March 2013; 433: 435. Disponible en http://www.nature.com/news/investigatingjournals-the-dark-side-of-publishing-1.12666

51. Moher D, Moher E. Stop Predatory Publishers Now: Act Collaboratively. Annals of Internal Medicine. Ann Intern Med 2 February 2016, doi:10.7326/M15-3015. Disponible en http://annals.org/article. aspx? articleid=2484878.

52. Jingfeng Xia J, Harmon JL, Connolly KG. Who Publishes in "Predatory" Journals?. Journal of the Association for Information Science and Technology 2015; 66 (7): 1406-1417. 